\title{
MORTALIDADE PERINATAL: UMA ANÁLISE COM ENFOQUE NA EVITABITABILIDADE
}

\author{
Jorge Otávio Maia Barretoํㅛ Inez Sampaio Nery², Yluska Myrna Meneses Brandão e Mendes ${ }^{3}$
}

\begin{abstract}
RESUMO: O estudo analisa a evitabilidade dos óbitos perinatais de Piripiri, Estado do Piauí, ocorridos em 2008 e sua importância para a composição da mortalidade infantil. Foram utilizados dados do Sistema de Informação sobre Mortalidade e do Sistema de Informação de Nascidos Vivos do Ministério da Saúde para a determinação dos coeficientes de mortalidade infantil, e analisadas as Declarações de Óbito e as fichas de investigação dos óbitos perinatais. Procedeuse à classificação segundo os critérios de evitabilidade de Wigglesworth que tem como parâmetros o momento do óbito, o peso ao nascer e a causa básica. Concluiu-se que, apesar do baixo Coeficiente de Mortalidade Infantil observado, a mortalidade perinatal ainda representa um desafio à redução da mortalidade infantil no município, verificando-se que $61,9 \%$ dos óbitos perinatais analisados apresentaram-se como evitáveis, suscitando a necessidade de ações de melhoria na atenção pré-natal, manejo obstétrico e atendimento ao neonato.
\end{abstract}

PALAVRAS-CHAVE: Mortalidade infantil; Mortalidade perinatal; Atestado de óbito; Causas de morte.

\section{PERINATAL MORTALITY: AN ANALYSIS FOCUSSING ON EVITABILITY}

\begin{abstract}
The study analyses the evitability of perinatal deaths in Piripiri in the state of Piauí in 2008 and considers its importance in the composition of infant mortality. Data for determining the coefficients of infant mortality were taken from the Ministry of Health's 'System for Information on Mortality' and 'System for Information on Live Births'. Death certificates and the files on perinatal deaths were also analysed. Data was grouped according to Wigglesworth's evitability classification system, whose parameters are time of death, birth weight and basic cause. It is concluded that, despite the low Infant Mortality coefficient observed, perinatal mortality still represents a challenge for efforts to reduce infant mortality in the county, considering that $61.9 \%$ of perinatal deaths analysed appeared to have been evitable. This should serve as a stimulus to improvement in prenatal care, obstetric management and neonatal care.
\end{abstract}

KEYWORDS: Infant mortality; Perinatal mortality; Death certificate; Causes of death.

\section{MORTALIDAD PERINATAL: UN ANÁLISIS CON ENFOQUE EN LA EVITABITABILIDAD}

RESUMEN: El estudio analiza la evitabilidad de los óbitos perinatales de Piripiri, estado de Piauí, ocurridos en 2008 y su importancia para la composición de la mortalidade infantil. Fueron utilizados datos del Sistema de Información sobre Mortalidad y del Sistema de Información de Nacidos Vivos del Ministerio de la Salud para determinación de los coeficientes de mortalidad infantil, y analizadas las Declaraciones de Óbito y las fichas de investigación de los óbitos perinatales. Se procedió a la clasificación de acuerdo a los criterios de evitabilidad de Wigglesworth, lo cual tiene como parámetros el momento del óbito, el peso al nacer y la causa básica. Se concluyó que, a pesar del bajo Coeficiente de Mortalidad Infantil observado, la mortalidad perinatal todavía representa un desafío a la reducción de la mortalidad infantil en el municipio, observándose que $61,9 \%$ de los óbitos perinatales analizados se presentaron como evitables, suscitando la necesidad de acciones de mejoría en la atención prenatal, manejo obstétrico y atendimiento al neonato.

PALABRAS CLAVE: Mortalidad infantil; Mortalidad perinatal; Atestado de óbito; Causas de muerte.

\footnotetext{
${ }^{1}$ Bacharel em Direito. Mestrando no Programa de Pós-Graduação em Políticas Públicas da Universidade Federal do Piauí - UFPI. Secretário Municipal de Saúde de Piripiri/PI, Secretário Extraordinário de Ciência e Tecnologia do Conselho Nacional de Secretarias Municipais de Saúde-CONASEMS.

${ }^{2}$ Enfermeira. Doutora em Enfermagem. Professora do Departamento de Enfermagem da UFPI.

${ }^{3}$ Enfermeira. Especialista em Saúde da Família. Coordenadora do SIM/SINASC do Departamento de Vigilância em Saúde da Secretaria Municipal de Saúde de Piripiri/PI.
}

\author{
Autor correspondente: \\ Jorge Otávio Maia Barreto \\ Universidade Federal do Piauí \\ Praça da Bandeira, 221 - 64260-000 Piripiri-PI-Brasil \\ E-mail: jorgeomaia@hotmail.com
}

Recebido: $15 / 09 / 10$ Aprovado: 15/02/11 


\section{INTRODUÇÃO}

No contexto das condições gerais de vida da população, que sofrem influência importante da saúde, o Coeficiente de Mortalidade Infantil (CMI), que mede o risco de morte de crianças menores de um ano de idade. É considerado um dos mais sensíveis indicadores de qualidade de vida de uma população ${ }^{(1-2)}$. É simples de ser calculado e reflete o estado de saúde da parcela mais vulnerável da população: os menores de um ano ${ }^{(3-4)}$

O CMI é, portanto, indicador importante para avaliar a qualidade de vida de uma população, indicando condições socioeconômicas e assistenciais às quais uma população está submetida, refletindo a qualidade da atenção à saúde nas ações de pré-natal, atenção ao parto e puerpério.

No Brasil, a mortalidade infantil apresentou uma importante redução nas duas últimas décadas, notadamente após a expansão da Estratégia Saúde da Família $^{(5)}$. Entretanto, esta redução ocorreu, principalmente, nos óbitos pós-neonatais, aqueles ocorridos após 26 dias de vida, enquanto o componente neonatal permaneceu com taxas elevadas, constituindo uma preocupação crescente para a saúde pública no Brasil.

O óbito perinatal, composto pelos óbitos fetais e neonatais precoces, representa maior dificuldade de redução, sendo esta mais lenta e complexa do que a mortalidade pós-neonatal e neonatal tardia. Pode-se considerar que, enquanto a mortalidade pós-neonatal configura-se num indicador que reflete a qualidade global de vida de uma população, a mortalidade perinatal indica a qualidade da assistência à gestante $\mathrm{e}$ ao neonato ${ }^{(6-7)}$.

Considera-se óbito fetal o natimorto com peso igual ou superior a 500 gramas e/ou com idade gestacional de 22 ou mais semanas de gestação. Óbito neonatal precoce é aquele ocorrido em crianças com até seis dias completos de vida ${ }^{(7)}$.

O coeficiente de mortalidade perinatal tem apresentado grandes diferenças entre as regiões brasileiras, o que aponta a necessidade da realização de estudos em nível regional e a aplicação de metodologias que enfoquem a evitabilidade dos óbitos perinatais, a fim de subsidiar o planejamento de estratégias que impactem o indicador ${ }^{(7-8)}$.

Considerando-se que o óbito fetal e o neonatal precoces guardam entre si etiologia assemelhada, especialmente nas faixas de peso ao nascer acima de 1.500 gramas, é possível analisar estes óbitos conjun- tamente, verificar se os mesmos são evitáveis e quais as determinantes das taxas de mortalidade perinatal não seguem a tendência de redução da mortalidade infantil(6)

Em Piripiri, Estado do Piauí, o Sistema de Informações sobre Mortalidade do Ministério da Saúde $(\mathrm{SIM})^{(9)}$ registrou, em 2000, 50 óbitos infantis, dos quais quatro foram óbitos neonatais tardios, 24 neonatais precoces e 22 pós-neonatais. Da relação de 1.363 nascidos vivos registrados no Sistema de Informações de Nascidos Vivos (SINASC) ${ }^{(10)}$, naquele ano, obteve-se o CMI de 36,68/1000. Já em 2008, o SIM registrou 15 óbitos infantis, sendo um neonatal tardio, 10 neonatais precoces e quatro pós-neonatais, em face de 928 nascidos vivos, com CMI de 16,16 por mil nascidos vivos, observando-se uma queda de $55,94 \%$ em relação a 2000 , o que situa o município no estrato de baixa mortalidade infantil ${ }^{(7)}$.

Entretanto, a mortalidade neonatal precoce não acompanhou essa redução. Observou-se o coeficiente de 17,60 por mil nascidos vivos em 2000 e de 10,78 em 2008, uma redução de apenas 38\%. Em 2000, o componente pós-neonatal representou $44 \%$ dos óbitos infantis em Piripiri, reduzindo, em 2008, a sua participação no total dos óbitos infantis para 26,6\%. Entre os anos de 2000 e 2008 a redução dos óbitos pós-neonatais em residentes de Piripiri foi de $81,8 \%$.

Esses números evidenciam que o óbito neonatal, especialmente o precoce, representa um importante desafio ao Sistema Único de Saúde (SUS), em Piripiri, para a redução da mortalidade infantil, requerendo análise da sua evitabilidade, a partir das informações disponíveis nas bases oficiais, a fim de subsidiar o planejamento de ações.

Por outro lado, poucos estudos no Brasil abordam, de forma específica, a análise regional da mortalidade perinatal, principalmente pela dificuldade de fidedignidade das informações. A investigação dos óbitos fetais e infantis representa a principal forma de buscar a validação e complementação das informações existentes no SIM, que utiliza informações do nível local, consolidadas em uma base nacional, produzindo dados em níveis de agregação municipal, estadual e federal. A investigação do óbito, em especial do perinatal, pode qualificar as informações já disponíveis ou acrescentar outras que não se encontram disponíveis no SIM; estas são coletadas das declarações de óbitos (e muitas vezes podem apresentar inconsistências ou indefinição), essenciais para a qualificação da discussão de ações de saúde mais eficazes para a redução da 
mortalidade infantil.

Assim, este estudo teve o objetivo de analisar os óbitos perinatais de residentes em Piripiri, ocorridos no ano de 2008, com foco na evitabilidade, a fim de estimar os óbitos reduzíveis e orientar ações para a melhoria da qualidade da assistência à gestante e ao neonato. Utilizou-se, para tanto, os critérios da classificação proposta por Wigglesworth ${ }^{(11)}$, e modificada por Keeling et al. ${ }^{(12)}$.

\section{METODOLOGIA}

Esse é um estudo retrospectivo, de abordagem quantitativa, sobre os óbitos perinatais de filhos de mulheres residentes em Piripiri, durante o ano de 2008, investigados pela Estratégia Saúde da Família (ESF) e Coordenação Municipal do SIM.

Os óbitos perinatais ocorridos em residentes no referido município, em 2008, foram analisados e classificados segundo o critério de evitabilidade de Wigglesworth ${ }^{(11)}$ que:

(...) propõe uma classificação simplificada de causas de óbito que prioriza o enfoque de evitabilidade. $\mathrm{O}$ autor aponta a necessidade de se proceder a avaliação da assistência perinatal em curso e propõe que as intervenções mais efetivas podem ser aquelas relativas às alterações de procedimentos de rotina nos serviços, e não necessariamente maiores gastos com tecnologia médica complexa. Esta metodologia proposta utiliza informações clínicas de fácil obtenção através da análise de prontuários, selecionando para análise os aspectos passíveis de intervenção pelos serviços ${ }^{(6: 762)}$.

Para fins de comparação, procedeu-se à análise de todos os óbitos infantis de residentes em Piripiri ocorridos nos anos de 2006 a 2008, utilizando, primeiramente, os espelhos das declarações de óbitos extraídos da base local do SIM, a fim de identificar a composição do óbito infantil naqueles anos. Foram considerados os seguintes componentes do óbito infantil: óbito fetal; óbito neonatal, subdividido em óbito neonatal precoce e tardio; óbito perinatal; e óbito pós-neonatal ${ }^{(7)}$.

Foram calculados, pelo método direto ${ }^{(7)}$, os coeficientes de mortalidade infantil e de seus componentes, a fim de identificar a composição da mortalidade infantil no referido município, no período de 2006 a 2008.

Como se verificou a prevalência dos óbitos neonatais precoces na composição da mortalidade infantil em Piripiri, nos anos analisados, especialmente em 2008, optou-se pela análise desse componente e dos óbitos fetais. Assim, ampliou-se análise para os óbitos perinatais, em face da aproximação da etiologia e das determinantes nas duas categorias ${ }^{(13)}$, restringindo-se ao ano de 2008, o qual apresentou o menor coeficiente de mortalidade perinatal da série, mas registrou a maior participação do óbito neonatal precoce na composição do óbito infantil (66,66\% em 2008, contra $64,70 \%$ em 2007 e $52,94 \%$ em 2006).

A análise incluiu os óbitos perinatais de residentes ocorridos também fora do município, por meio do espelho da declaração de óbito fornecido pela base local do SIM.

Para analisar a evitabilidade dos óbitos, utilizouse a classificação de Wigglesworth ${ }^{(11)}$, modificada por Keeling et al. ${ }^{(13)}$, recomendada para este tipo de análise e focada no potencial de evitabilidade dos óbitos perinatais e sua relação com a qualidade da assistência ${ }^{(6)}$. Os óbitos perinatais foram alocados em cinco categorias mutuamente excludentes, de base fisiopatológica e associadas a possíveis falhas na intervenção assistencial. Para avaliar as causas básicas, priorizou-se o evento inicial na cadeia causal, buscando morbidades que tivessem desencadeado o desfecho reprodutivo. As informações das declarações de óbito foram complementadas pela análise das fichas de investigação de óbito preenchidas à época da ocorrência pela equipe da ESF.

As informações básicas sobre os nascidos vivos foram obtidas a partir do SINASC, base local, retroalimentada pela base nacional, da SMS de Piripiri. O banco de dados do SIM, base local, foi utilizado para impressão dos espelhos das declarações de óbitos infantis nos anos analisados.

Os óbitos perinatais investigados foram estratificados por peso ao nascer e as causas de morte analisadas, segundo os critérios de evitabilidade adotados e referidos acima.

Quando se verificou divergência entre as informações existentes na ficha de investigação de óbito e as apresentadas na declaração de óbito, especialmente quanto ao momento do óbito - se antes, durante ou após o parto - optou-se por considerar o registro da ficha de investigação.

\section{RESULTADOS}

Na tabela 1, são apresentados os resultados obtidos a partir dos dados do SIM e SINASC relativos aos nascimentos, óbitos infantis e fetais, bem como os respectivos coeficientes de mortalidade por componente etário. 
Tabela 1 - Mortalidade infantil e fetal por componentes etários. Piripiri-PI, 2006 a 2008

\begin{tabular}{lccc}
\hline & $\mathbf{2 0 0 6}$ & $\mathbf{2 0 0 7}$ & $\mathbf{2 0 0 8}$ \\
\hline População residente & 62.291 & 62.616 & 61.975 \\
Nascidos vivos & 987 & 906 & 928 \\
Óbitos infantis & 17 & 17 & 15 \\
Coeficiente de mortalidade infantil* & 17,22 & 18,76 & 16,16 \\
Óbitos fetais & 18 & 18 & 11 \\
Coeficiente de mortalidade fetal* & 18,24 & 19,87 & 11,85 \\
Óbitos neonatais precoces & 9 & 11 & 10 \\
Coeficiente de mortalidade neonatal precoce* & 9,12 & 12,14 & 10,78 \\
Óbitos neonatais tardios & 3 & 4 & 1 \\
Coeficiente de mortalidade neonatal tardia* & 3,04 & 4,42 & 1,08 \\
Óbitos pós-neonatais & 5 & 2 & 4 \\
Coeficiente de mortalidade pós-neonatal* & 5,07 & 2,21 & 4,31 \\
Coeficiente de mortalidade perinatal & 26,87 & 31,39 & 22,36 \\
\end{tabular}

Fonte: SIM/SINASC SMS Piripiri-PI

* Por mil nascidos vivos

Os dados do SINASC indicam que, entre 2006 e 2008, os nascimentos de residentes em Piripiri apresentaram queda pouco importante $(5,97 \%)$, com números absolutos de 987 (2006), 906 (2007) e 928 (2008). Observou-se redução no número absoluto de óbitos perinatais, registrando-se a seguinte sequência: 27 (2006), 29 (2007) e 21 (2008), com variação de 25\% no período.

O percentual de óbitos fetais superou o de óbitos neonatais em todo o período, apesar de se verificar uma redução de 18 (2006), 18 (2007) e 11 (2008), observando-se uma redução de $38 \%$. Dessa forma, houve uma redução no coeficiente de mortalidade perinatal, declinando de 26,87 para 22,36, entre 2006 e 2008.

Em 2008, foram registrados no SIM 15 óbitos infantis e 11 óbitos fetais de residentes. Destes, todos foram investigados e ficaram assim distribuídos: 11 fetais e 10 neonatais precoces, totalizando 21 óbitos perinatais. Ao se comparar os percentuais de óbitos fetais e neonatais precoces na composição dos óbitos perinatais em Piripiri, no ano de 2008, observou-se equilíbrio entre o percentual de óbitos fetais (52\%) e neonatais precoces $(48 \%)$.

O coeficiente de mortalidade perinatal em 2008 foi de 22,36 por mil nascidos (vivos e natimortos), representando uma taxa de mortalidade superior em
$38 \%$ se comparada à taxa de mortalidade infantil geral. O coeficiente de mortalidade fetal em $2008(11,85$ por mil nascidos vivos e natimortos) foi inferior ao de mortalidade infantil ( 16,16 por mil nascidos vivos) e ligeiramente superior ao da mortalidade precoce (10,78 por mil nascidos vivos).

A tabela 2 mostra que um percentual importante dos óbitos perinatais $(38,1 \%)$ foi classificado como de natimortos (anteparto). Verificou-se também a importância do percentual de asfixia durante o trabalho de parto $(26,8 \%)$.

Na tabela 2, também está representada a distribuição dos óbitos por faixa de peso ao nascer e classificados pelos critérios de Wigglesworth ${ }^{(11)}$. Observa-se a concentração de óbitos perinatais nas faixas de peso ao nascer superior a $1.000 \mathrm{~g}$. Na análise dos óbitos perinatais de residentes no referido município, no ano de 2008 , encontrou-se $61,9 \%(n=13)$ situados nas faixas de peso acima de $1.000 \mathrm{~g}$. Se incluirmos as malformações congênitas registradas em óbitos nas faixas de peso acima de $1.000 \mathrm{~g}$, o percentual de óbitos em faixas de peso viáveis sobe para 76,19\% $(\mathrm{n}=16)$.

Não se observou, dentre os óbitos analisados, o registro de causas específicas do grupo 5 da classificação de Wigglesworth ${ }^{(1)}$, as quais estão relacionadas com infecções decorrentes de falhas na isoimunização obstétrica ou neonatal. 
Tabela 2 - Óbitos perinatais por faixa de peso ao nascer, segundo a classificação de Wigglesworth, adaptada por Keeling et al. Piripiri-PI, 2008

\begin{tabular}{|c|c|c|c|c|c|c|c|c|c|c|}
\hline \multirow[t]{2}{*}{$\begin{array}{l}\text { Peso ao nascer } \\
\text { (gramas) }\end{array}$} & \multicolumn{2}{|c|}{$\begin{array}{l}\text { Anteparto } \\
\text { (Grupo 1) }\end{array}$} & \multicolumn{2}{|c|}{$\begin{array}{l}\text { Malformação } \\
\text { (Grupo 2) }\end{array}$} & \multicolumn{2}{|c|}{$\begin{array}{l}\text { Prematuridade } \\
\quad \text { (Grupo 3) }\end{array}$} & \multicolumn{2}{|c|}{$\begin{array}{l}\text { Asfixia intraparto } \\
\quad \text { (Grupo 4) }\end{array}$} & \multicolumn{2}{|c|}{$\begin{array}{l}\text { Específicas } \\
\text { (Grupo 5) }\end{array}$} \\
\hline & $\mathrm{n}$ & $\%$ & $\mathrm{n}$ & $\%$ & $\mathrm{n}$ & $\%$ & $\mathrm{n}$ & $\%$ & $\mathrm{n}$ & $\%$ \\
\hline $500-999$ & 02 & $9,5 \%$ & - & - & 03 & $14,2 \%$ & - & - & - & - \\
\hline $1000-1499$ & 01 & $4,8 \%$ & - & - & - & - & 02 & $9,5 \%$ & - & - \\
\hline $1500-2500$ & 02 & $9,5 \%$ & 02 & $9,5 \%$ & 01 & $4,8 \%$ & - & - & - & - \\
\hline $2500-2999$ & 01 & $4,8 \%$ & $\%$ & - & - & - & 02 & $9,5 \%$ & - & - \\
\hline$\geq 3000$ & 02 & $9,5 \%$ & 01 & $4,8 \%$ & - & - & 02 & $9,5 \%$ & - & - \\
\hline Total & 08 & $38,1^{\circ}$ & 03 & $14,3 \%$ & 04 & $19 \%$ & 06 & $28,6 \%$ & - & - \\
\hline \multicolumn{11}{|c|}{$\begin{array}{l}\text { Na tabela 3, são apresentados os coeficientes } \\
\text { de mortalidade fetal, neonatal precoce e perinatal } \\
\text { precoce por faixa de peso ao nascer. } \\
\text { Os coeficientes de mortalidade por faixas de }\end{array}$} \\
\hline & & & \multicolumn{6}{|c|}{ Peso ao nascer (gramas) } & & Total \\
\hline & & & 500-999 & \multicolumn{2}{|c|}{$1000-1499$} & \multicolumn{2}{|c|}{$1500-2500$} & $\geq 2500$ & & \\
\hline \multicolumn{3}{|c|}{$\begin{array}{l}\text { Nascidos vivos de residente na faixa } \\
\text { de peso ao nascer }\end{array}$} & 4 & \multicolumn{2}{|c|}{7} & \multicolumn{2}{|c|}{39} & 878 & & 928 \\
\hline \multicolumn{3}{|c|}{ Óbitos fetais } & 2 & \multicolumn{2}{|c|}{2} & \multicolumn{2}{|c|}{2} & 5 & & 11 \\
\hline \multicolumn{3}{|c|}{$\begin{array}{l}\text { Coeficiente da mortalidade fetal por } \\
\text { faixa de peso ao nascer* }\end{array}$} & 500,00 & \multicolumn{2}{|c|}{285,71} & \multicolumn{2}{|c|}{51,28} & 5,69 & & - \\
\hline \multicolumn{3}{|c|}{ Óbitos neonatais precoces } & 3 & \multicolumn{2}{|c|}{2} & \multicolumn{2}{|l|}{2} & 3 & & 11 \\
\hline \multicolumn{3}{|c|}{$\begin{array}{l}\text { Coeficiente de mortalidade neonatal } \\
\text { precoce por faixa de peso ao nascer* }\end{array}$} & 750,00 & \multicolumn{2}{|c|}{285,71} & \multicolumn{2}{|c|}{51,28} & 3,42 & & - \\
\hline \multicolumn{3}{|l|}{ Óbitos perinatais } & 5 & & 4 & \multicolumn{2}{|l|}{4} & 8 & & 21 \\
\hline \multicolumn{3}{|c|}{$\begin{array}{l}\text { Coeficiente de mortalidade perinatal } \\
\text { por faixa de peso ao nascer }\end{array}$} & 833,33 & & 4,44 & \multicolumn{2}{|c|}{97,56} & 9,06 & & - \\
\hline
\end{tabular}

Fonte: Ministério da Saúde, SIM/SINASC, 2009

de peso de 500 a 999 gramas encontrado foi de 833,33 por mil nascidos. A faixa de peso seguinte, que compreende de 1.000 a 1.499 gramas, também apresentou alta mortalidade, com coeficiente de 444,44 óbitos por mil nascidos vivos.

Da mesma maneira, nas faixas de peso de 1.500 a 2.500 gramas, encontrou-se alta mortalidade perinatal $(97,56$ por mil nascidos vivos na faixa de peso), com distribuição equivalente entre os componentes neonatal precoce e fetal.

Por fim, observou-se que $38,09 \%$ dos óbitos perinatais ocorreram em crianças com peso adequado ao nascimento, ou seja, acima de 2.500 gramas.

Passando-se à análise da evitabilidade, a tabela 4 apresenta a classificação dos óbitos perinatais por faixa de peso ao nascer, após a classificação segundo os critérios de Wigglesworth ${ }^{(11)}$.

Destaca-se que, na primeira faixa, tendo ponte de corte o peso ao nascer maior que 1.000 gramas, o percentual de óbitos com causas evitáveis encontrado foi $61,9 \%$. 
Tabela 4 - Evitabilidade dos óbitos perinatais por peso ao nascer. Piripiri-PI, 2008

\begin{tabular}{cccc}
\hline Total de óbitos & Peso ao nascer & $\begin{array}{c}\text { Óbitos } \\
\text { evitáveis }\end{array}$ & $\%$ \\
\hline 21 & $>1000 \mathrm{~g}$ & 13 & $61,9 \%$ \\
21 & $>1500 \mathrm{~g}$ & 10 & $47,6 \%$ \\
21 & $>2500 \mathrm{~g}$ & 7 & $33,3 \%$ \\
\hline
\end{tabular}

\section{DISCUSSÃO}

Os resultados sugerem que a maior parte dos óbitos perinatais ocorridos em Piripiri, em 2008, eram evitáveis. Os dois principais grupos da classificação Wigglesworth ${ }^{(11)}$ encontrados no município, grupos 1 e 4, estão relacionados com a necessidade de melhoria da assistência dispensada à gestante durante o período pré-natal, pré-parto, e, especialmente, durante o parto. Os resultados refletem falhas na atenção pré-natal, além de condições maternas adversas, mas também indicam problemas no manejo obstétrico e neonatal, especialmente em face do elevado percentual de asfixia durante o trabalho de parto $(28,6 \%)$. É importante ressaltar que a asfixia intraparto, quando não causa $o$ óbito, produz sequelas graves, com comprometimentos neurológicos e funcionais, o que afeta a qualidade de vida da criança e representa um alto custo social.

A imaturidade do concepto, por sua vez, se apresenta também como outra causa relevante de óbito perinatal (19\%) e também expressa falhas no manejo obstétrico e/ou deficiências no atendimento ao recém nascido no berçário, além de repercutir falhas no prénatal.

Os resultados sugerem especial dificuldade do serviço de assistência ao parto em lidar com o baixo peso extremo ao nascer, uma vez que o óbito perinatal em nascidos vivos na faixa de peso de 500 a 999 gramas é exageradamente alto: 833,33 por mil nascidos naquela faixa de peso. $\mathrm{O}$ mesmo ocorre na faixa de peso ao nascer seguinte, de 1.000 a 1.499 gramas, com 444,44 por mil nascidos vivos, contrariando a viabilidade de crianças nascidas com peso superior a 1.000 gramas. A alta mortalidade encontrada expõe falhas na assistência ao parto e ao neonato de baixo peso.

Assim, considerando-se que a mortalidade perinatal entre crianças com baixo peso (abaixo de 2.500 gramas) apresentou-se bastante elevada se comparada com a mortalidade infantil geral, ou mesmo com a taxa de mortalidade perinatal, constatou-se a necessidade do aprimoramento na assistência ao parto e ao neonato de baixo peso no âmbito da assistência hospitalar, bem como de melhoria na detecção, no pré-natal, das situações de risco que incidam sobre o nascimento de crianças com baixo peso.

Não há dúvida de que o baixo peso ao nascer e a prematuridade são relevantes fatores de risco para o desfecho reprodutivo desfavorável, representando o fator mais comumente associado com a mortalidade infanti $1^{(14)}$. Entretanto, o reconhecimento da fragilidade do neonato de baixo peso é determinante para um tratamento diferenciado, a fim de que se possam implementar ações que garantam a viabilidade de crianças que estejam abaixo de 2.500 gramas no nascimento.

Por outro lado, o alto percentual $(38,09 \%)$ de óbitos perinatais em crianças com peso adequado ao nascimento reforça a necessidade de aprimoramento da assistência ao parto e ao neonato.

De forma geral, os resultados apontam que as mortes ocorridas em crianças com peso superior a 1.000 gramas se concentraram em dois grupos quanto à evitabilidade. O primeiro está relacionado com a adequabilidade e qualidade da atenção pré-natal, e o segundo, à qualidade da atenção obstétrica e neonatal.

A presente análise apontou a necessidade de implementar opções para a melhoria da atenção à gestante, ao parto e ao neonato. Tais intervenções podem consistir em ações de baixo custo e com efetivo impacto sobre a qualidade da assistência materna e ao recém-nascido, tais como qualificação permanente dos profissionais na atenção pré-natal, manejo obstétrico e atendimento ao recém-nascido na sala de parto $^{(15-16)}$.

Outras intervenções relacionadas aos arranjos organizacionais do serviço de atenção ao parto e ao neonato, tais como a ampliação e melhoria da atuação da equipe de enfermagem na assistência maternoinfantil, também podem proporcionar melhores resultados na superação de momentos críticos no parto e no cuidado neonatal do prematuro $^{(17)}$.

\section{CONSIDERAÇÕES FINAIS}

Neste estudo, observou-se que a mortalidade infantil em Piripiri, no ano de 2008, foi composta na sua maioria por óbitos neonatais precoces e, embora tenha havido um declínio nas taxas de mortalidade infantil e perinatal, a situação ainda representa uma 
preocupação para a gestão local do SUS. O óbito neonatal precoce deve ser abordado juntamente com o óbito fetal, em face de suas similaridades especialmente nas faixas de peso ao nascer acima de 1.000 gramas.

Constatou-se que a maior parte dos óbitos ocorreu por causas evitáveis, podendo ser enfrentadas, principalmente por meio de intervenções que busquem alterações de procedimentos de rotina nos serviços, que não implicam necessariamente em gastos com tecnologia médica avançada.

A predominância de óbitos perinatais na composição do coeficiente de mortalidade infantil do $\mathrm{Mu}-$ nicípio de Piripiri merece especial atenção, já que a mortalidade infantil pós-neonatal apresentou grande redução entre 2000 e 2008, possivelmente em função da ampliação do acesso aos serviços de atenção primária desenvolvidos pela ESF no município.

A qualidade da informação é essencial para a tomada de decisão quanto às ações a serem adotadas. No caso desta análise, a SMS de Piripiri demonstrou importante melhoria do registro do óbito, especialmente o infantil e fetal. Entretanto, é possível ampliar a capacidade de coleta de informações que conduzam a uma análise cada vez mais próxima da realidade dos óbitos perinatais, em especial dos óbitos fetais. A investigação do óbito infantil é uma importante estratégia para o planejamento da assistência à saúde e redução da mortalidade infantil.

Apesar dos avanços na redução da mortalidade infantil no município, a evitabilidade dos óbitos analisados ainda coloca a mortalidade perinatal como um desafio a ser enfrentado no âmbito da atenção à gestante e assistência hospitalar. Ênfase deve ser dada à implantação de rotinas voltadas para o parto de risco, desde o pré-natal à assistência hospitalar, especialmente com a garantia da assistência humanizada e competente.

O critério de classificação da evitabilidade dos óbitos infantis e fetais objetiva identificar causas que podem ser reduzidas mediante ações simples e de baixo custo. Os resultados desta análise sugerem que são necessárias iniciativas, em especial para a melhoria da assistência ao parto e ao neonato, principalmente mediante a implantação de rotinas e fluxos que garantam a adequada assistência ao parto, ainda mais quando se configure o risco para a mãe e a criança, não representando necessariamente dependência de investimento em tecnologia.

Por fim, a gestão local do SUS deve promover a discussão ampliada do problema, a fim de que haja o envolvimento ativo dos setores implicados na busca por soluções que enfrentem o problema da mortalidade infantil por meio da redução da mortalidade perinatal.

\section{REFERÊNCIAS}

1. Vermelho LL, Leal AJC, Kale PL. Indicadores de saúde. In: Medronho R, editor. Epidemiologia. São Paulo: Atheneu; 2006.

2. Tanaka ACA, Siqueira AAF, Bafile PN. Situação de saúde materna e perinatal no Estado de São Paulo, Brasil. Rev Saúde Pública. 1989;23(1):67-75.

3. Duarte CMR. Reflexos das políticas de saúde sobre as tendências da mortalidade infantil no Brasil: revisão da literatura sobre a última década. Cad Saúde Pública. 2007;23(7):1511-28.

4. Bezerra Filho JG, Kerr-Pontes LRS, Barreto ML. Mortalidade infantil no Ceará. Rev Bras Saúde Mater Infant. 2007;7(2):135-42.

5. Macinko J, Guanais FC, Souza MFM. An evaluation of impact of the family health program on infant mortality in Brazil, 1990-2002. J Epidemiol Comunity Health. 2006;60(1):13-9.

6. Lansky S, França E, Leal MC. Mortalidade perinatal e evitabilidade: revisão da literatura. Rev Saúde Pública. 2002;36(6):759-72.

7. Ministério da Saúde (BR). Manual dos comitês de prevenção do óbito infantil e fetal. Brasília: Ministério da Saúde; 2005.

8. Fonseca SC, Coutinho ESF. Características biológicas e evitabilidade de óbitos perinatais em uma localidade na cidade do Rio de Janeiro, 1999 a 2003. Rev Bras Saúde Mater Infant. 2008;8(2):171-8.

9. Ministério da Saúde (BR). Departamento de Informática do SUS/Informações de Saúde/Estatísticas Vitais/ Sistema de Informações sobre Mortalidade (SIM). [acesso em 05 mai 2009]. Disponível: http://www2.datasus.gov.br/ DATASUS/index. php?area $=0205$

10. Ministério da Saúde (BR) Departamento de Informática do SUS/Informações de Saúde/Estatísticas Vitais/ Sistema de Informações de Nascidos Vivos (SINASC). [acesso em 05 mai 2009] Disponível: http://www2. datasus.gov.br/DATASUS/index.php?area=0205 
11. Wigglesworth JS. Monitoring perinatal mortality: a pathophysiological approach. Lancet. 1980;2(8196):684-6.

12. Keeling JW, MacGillivray I, Golding J, Wigglesworth J, Berry J, Dunn PM. Classification of perinatal death. Arch Dis Child. 1989;64(10):1345-51.

13. Rouquayrol MZ, Correia LL, Barbosa LMM, Xavier LGM, Oliveira JWO, Fonseca W. Fatores de risco de natimortalidade em Fortaleza: um estudo caso controle. J Pediatr. 1996;72(6):374-8.

14. Barros FC, Victora CG, Vaughan JP. Causas de mortalidade perinatal em Pelotas, RS (Brasil). Utilização de uma classificação simplificada. Rev Saúde Pública. 1987;21(2):310-6.

15. Caldeira AP, França E, Perpétuo IHO. Evolução da mortalidade infantil por causas evitáveis, Belo Horizonte, 1984-1998. Rev Saúde Pública. 2005;39(1):67-74.

16. Victora GC, Barros FC. Infant mortality due to perinatal causes in Brazil: trends, regional patterns and possible interventions. Med J. 2001;119(1):33-42.

17. Schmidt KT, Mello FT, Rosseto EG, Souza SNDH. Avaliação da assistência de enfermagem em unidade neonatal na perspectiva dos pais. Cogitare Enferm. 2010;15(3):460-6. 